\title{
HOXA9 is Underexpressed in Cervical Cancer Cells and its Restoration Decreases Proliferation, Migration and Expression of Epithelial-to-Mesenchymal Transition Genes
}

\author{
Liliana Alvarado-Ruiz ${ }^{1,2}$, Maria Guadalupe Martinez-Silva ${ }^{3}$, Luis Alberto \\ Torres-Reyes ${ }^{1,2}$, Patricia Piña-Sanchez ${ }^{4}$, Pablo Ortiz-Lazareno ${ }^{2}$, Alejandro Bravo- \\ Cuellar $^{2}$, Adriana Aguilar-Lemarroy ${ }^{2}$, Luis Felipe Jave-Suarez ${ }^{2 *}$
}

\begin{abstract}
HOX transcription factors are evolutionarily conserved in many different species and are involved in important cellular processes such as morphogenesis, differentiation, and proliferation. They have also recently been implicated in carcinogenesis, but their precise role in cancer, especially in cervical cancer (CC), remains unclear. In this work, using microarray assays followed by the quantitative polymerase chain reaction (qPCR), we found that the expression of $25 \mathrm{HOX}$ genes was downregulated in $\mathrm{CC}$ derived cell lines compared with nontumorigenic keratinocytes. In particular, the expression of $\mathrm{HOXA9}$ was observed as down-modulated in CCderived cell lines. The expression of $\mathrm{HOXA9}$ has not been previously reported in $\mathrm{CC}$, or in normal keratinocytes of the cervix. We found that normal $\mathrm{CC}$ from women without cervical lesions express $\mathrm{HOXA9}$; in contrast, $\mathrm{CC}$ cell lines and samples of biopsies from women with $\mathrm{CC}$ showed significantly diminished HOXA9 expression. Furthermore, we found that methylation at the first exon of $\mathrm{HOXA9}$ could play an important role in modulating the expression of this gene. Exogenous restoration of $\mathrm{HOXA9}$ expression in $\mathrm{CC}$ cell lines decreased cell proliferation and migration, and induced an epithelial-like phenotype. Interestingly, the silencing of human papilloma virus (HPV) E6 and E7 oncogenes induced expression of HOXA9. In conclusion, controlling HOXA9 expression appears to be a necessary step during $\mathrm{CC}$ development. Further studies are needed to delineate the role of $\mathrm{HOXA9}$ during malignant progression and to afford more insights into the relationship between downmodulation of HOXA9 and viral HPV oncoprotein expression during cercical cancer development.
\end{abstract}

Keywords: Cervical cancer - HOX genes - HOXA9 - proliferation - migration - epithelial-mesenchymal transition

Asian Pac J Cancer Prev, 17 (3), 1037-1047

\section{Introduction}

Cervical Cancer (CC) is the fourth most common cancer in women, and the seventh overall, with an estimated 528,000 new cases in 2012 (Ferlay et al., 2015). Development of this disease is closely associated with human papilloma virus (HPV) infection (Bosch and Munoz, 2002); however, it is estimated that only $0.03 \%$ of women who become infected with HPV proceed to develop CC (Sasagawa et al., 2012). Therefore, viral infection per se is not considered sufficient to generate a neoplastic process; it is, rather, a first alteration that predisposes cells to subsequent changes; if those do occur, the neoplasm is generated (Perez-Plasencia et al., 2008). It is evident that additional factors, such as genomic instability caused probably by viral oncogenes (E6 and E7), environmental factors, or the genetic background itself, are necessary for cervical tumorigenesis (Hyland et al., 2011). Alterations in developmental signaling pathways or transcription factors that regulate the ontogeny, such $H O X$ genes, have recently been proposed as alternative secondary modifications that could lead to malignant transformation (Karamboulas and Ailles, 2013).

$H O X$ genes in humans comprise a highly conserved family of 39 transcription factors that are grouped into four clusters: $H O X A ; B ; C$, and $D$, each group consisting of 13 paralog genes, with 9-11 members, distributed on the basis of sequence similarity and relative position within the group (Acampora et al., 1989). HOX genes play an important role in the delicate balance between cell proliferation and differentiation that is essential for normal fetal development during embryogenesis; the abnormal 
function of these transcription factors has been implicated in human diseases, particularly in neoplastic processes (Castelli-Gair, 1998; Lappin et al., 2006). Thus, in recent years, much effort has been devoted to the study of $H O X$ genes and proteins and their link with cancer (Shah and Sukumar, 2010). In cervix, despite some evidences of HOX member modulation (Alami et al., 1999; Hung et al., 2003; Lopez et al., 2006b; Gonzalez-Herrera et al., 2015), the participation of these transcription factors in cervical carcinogenesis has not been explored in depth. In this work, we evaluated $H O X$ gene deregulation in $\mathrm{CC}$; in particular, we focused on HOXA9 modulation and whether this phenomenon results in an advantage for the CC cell.

\section{Materials and Methods}

\section{Cell culture}

HeLa, SiHa, and C-33A cell lines derived from CC, as well as the non-tumorigenic keratinocyte $\mathrm{HaCaT}$ cell line, were obtained from the generosity of the German Cancer Research Center (DKFZ, Heidelberg, Germany). Cells were cultured in Dulbecco's Modified Eagle's Medium (DMEM) containing GlutaMAXTM and supplemented with $10 \%$ Fetal Bovine Serum (FBS), 100 $\mathrm{U} / \mathrm{ml}$ Penicillin, and $100 \mu \mathrm{g} / \mathrm{ml}$ Streptomycin, at $37^{\circ} \mathrm{C}$ with an atmosphere of 5\% CO2 and 90\% relative humidity (all of the previously mentioned products were acquired from GIBCO ${ }^{\circledR}$, Thermo Fisher Scientific, Inc., Waltham, MA, USA). Cells were propagated according to the methods recommended by the suppliers.

\section{Cervical samples and HPV detection}

Informed consent was obtained from all individual participants included in the study (Prot.R-2012-785-090). Patients were recruited at the Western Medical Center of the Mexican Institute of Social Security (IMSS) and Cervical scrapes of clinically healthy women without HPV infection were collected with a cytobrush during gynecological examination and placed into PreservCyt transport medium (Hologic, Bedford, MA, USA). Cervical biopsies of patients diagnosed with Squamous Cell Carcinoma (SCC) and with HPV infection were collected, frozen, and stored until processing. HPV detection was performed as follows: genomic DNA was extracted from all samples and screened by conventional PCR utilizing the PGMY 09/11 primers, as described elsewhere (Gravitt et al., 2000), and samples that were positive to HPV were further analyzed using the Linear Array HPV Genotyping Test (Roche Applied Science, Penzberg, Germany) (Coutlee et al., 2006).

\section{Isolation of primary keratinocytes}

Primary Keratinocytes (Kers) were obtained from the normal cervixes of women who underwent hysterectomy, and the samples were taken under signed informed consent at the Western Medical Center - IMSS. Biopsies were collected in EPILIFE CF medium (Thermo Fisher Scientific, Inc.) with $100 \mathrm{U} / \mathrm{ml}$ Penicillin and $100 \mu \mathrm{g} /$ $\mathrm{ml}$ Streptomycin. After removal of connective and fatty tissue, the biopsy was chopped into $0.5-\mathrm{cm} 2$ fragments and treated with $25 \mathrm{U} / \mathrm{ml}$ collagenase (Sigma-Aldrich
Quimica, S. A. de R.L. de C.V, Toluca, México) and 25 $\mathrm{U} / \mathrm{ml}$ dispase (Thermo Fisher Scientific, Inc.) overnight at $4 \mathrm{oC}$ with constant movement. Then, the epidermis was removed from connective tissue with tweezers. Epithelial cells were disrupted by incubation for $30 \mathrm{~min}$ at $37^{\circ} \mathrm{C}$ in $2 \mathrm{ml}$ of $0.25 \%$ trypsin-EDTA solution (Thermo Fisher Scientific, Inc.). Trypsin was neutralized with FBS and the cells were collected by centrifugation and resuspended in $13 \mathrm{ml}$ of selective medium for keratinocytes EPILIFE CF supplemented with Human Keratinocyte Growth Supplement containing: $0.2 \% \mathrm{v} / \mathrm{v}$ pituitary gland extract (BPE), $5 \mu \mathrm{g} / \mathrm{ml}$ bovine insulin, $0.18 \mu \mathrm{g} / \mathrm{ml}$ hydrocortisone, $5 \mu \mathrm{g} / \mathrm{ml}$ bovine transferrin, $0.2 \mathrm{ng} / \mathrm{ml}$ human Epidermal Growth Factor (EGF), and Gentamicin/Amphotericin (all from Thermo Fisher Scientific, Inc.). The cultures were maintained at $37^{\circ} \mathrm{C}$ in a humidified atmosphere with $5 \%$ $\mathrm{CO}_{2}$.

\section{RNA isolation and expression microarrays}

Total RNA from the different cell lines, cervical scrapes, and biopsies was extracted with the NucleoSpin RNA Kit (Macherey-Nagel GmbH \& Co. KG, Düren, Germany) according to the manufacturer's instructions. RNA was quantified by absorbance at 260/280 nm. For microarray analysis, RNA quality was determined using an Agilent 2100 Bioanalyzer and the RNA 6000 NanoChip Kit (Agilent Technologies, Santa Clara, CA, USA). Double stranded complementary DNA (cDNA) was generated from $10 \mu \mathrm{g}$ of RNA using the cDNA Synthesis Kit System (Roche Applied Science) and purified with the GenEluteTM PCR Clean-Up Kit (Sigma-Aldrich Quimica, S. A. de R.L. de C.V.); thereafter, the cDNA was labeled with $\mathrm{Cy} 3$ and hybridized in a Human Gene Expression Array 12x135K (Roche Applied Science). Microarrays were scanned on the MS200 Scanner and the data obtained was processed using DEVA ver 1.2 software (Roche Applied Science). Fluorescence intensities were normalized using the RMA algorithm and the information was subsequently analyzed with CLC Main WorkBench ver. 7.0.3 software. Genes that were differentially expressed with a fold-change $>1.5$ and that exhibited a permutation $\mathrm{p}$-value $<0.05$ were selected.

\section{RT-PCR and $q P C R$ assays}

cDNA synthesis was performed from $5 \mu \mathrm{g}$ of total RNA using the Transcriptor First Strand cDNA Synthesis Kit (Roche Applied Science) primed with Oligo dT. The cDNA obtained was employed in a quantitative Polymerase Chain Reaction (qPCR) to evaluate gene expression levels utilizing the LightCycler ${ }^{\circledR}$ FastStart DNA Master PLUS SYBR Green I Kit with 2.0 LightCycler technology (Roche Applied Science) under conditions specified by the manufacturer. The gene-specific primers (Supplementary Table 1) were designed with Oligo Primer Analysis ver. 6.0 software (Molecular Biology Insights, Inc., Colorado Springs, CO, USA) from sequences obtained at the Entrez Nucleotide Database of the National Center of Biotechnology Information (NCBI). Quantification of PCR products was calculated employing Light Cycler ver. 4.1 software and was calculated using the following reference genes: GAPDH; ACTB; RPLP0, and RP18S. 
Two types of analysis were used: $2 \Delta \Delta \mathrm{CP}$ and $\Delta \mathrm{CP}$. A melting curve analysis was also performed for each gene to determine the specificity of the reaction.

\section{CpG methylation detection assay}

To determine the presence of $\mathrm{CpG}$ islands, analysis of 1,400-bp upstream and 800-bp downstream of translation start was performed using the MethPrimer online tool (Li and Dahiya, 2002). Additionally, localization of restriction sites for $\mathrm{MspI}$ and HpaII restriction enzymes was determined utilizing CLC Main Workbench ver. 7.0 software (Qiagen, Germantown, MD, USA). Specific primer pairs were designed using Oligo Primer Analysis ver. 6.0 software (Molecular Biology Insights, Inc.) to amplify five different zones (Z1-Z5), which cover the different $\mathrm{CpG}$ islands found (Supplementary Table 2, Figure 3a). Genomic DNA from cell lines and primary keratinocytes was extracted with the QIAamp DNA Mini Kit (Qiagen) and digested with MspI restriction enzyme, and its isoschizomer non-methylation-sensitive HpaII (New England BioLabs, Inc., Ipswich, MA, USA) at $37^{\circ} \mathrm{C}$ for $1 \mathrm{~h}$. The digested DNA was used as template for PCR amplification reactions utilizing the Expand High Fidelity enzyme (Sigma-Aldrich Quimica S. de R.L. de C.V.) as recommended by the manufacturer. The amplification products were separated by electrophoresis in $2 \%$ agarose gels, visualized, and digitalized for analysis employing a DigiDoc-It system program (UVP, LLC. Upland, CA, USA).

\section{Immunohistochemistry}

Histologically normal cervical epithelium (35) and CC (21) samples that were paraffin-embedded were utilized to construct a Tissue MicroArray (TMA) using the Tissue Microarray ATA 100 (Chemicon, Temecula, CA, USA). TMA slices were used for automated immunodetection assays utilizing the Ventana BenchMark System (Roche Applied Science), the anti-HOXA9 antibody (cat. Ab922565; Abcam, Cambridge, MA, USA), and the ultraView Universal DAB Detection Kit (Roche Applied Science). HOXA9 expression was evaluated by a Pathologist and classified according to level of positivity as low, medium, or high.

\section{Plasmid constructs}

The commercial HOXA9 Open Reading Frame (ORF) (cDNA clone MGC: 19648; Thermo Fisher Scientific, Inc. ) was subcloned into the pLVX-tight-Puro vector, a tetracycline-inducible expression system, and into the pLVX-Puro vector, a constitutive expression system (Takara Bio, Inc., Kusatsu, Shiga, Japan). Lentiviral particles were produced by transfecting Lenti-X ${ }^{\text {TM }} 293 \mathrm{~T}$ cells with Lipofectamine 2000 (Thermo Fisher Scientific, Inc.) and Lenti- $\mathrm{X}^{\mathrm{Tм}}$ HT Packaging Systems (Takara Bio, Inc.), according to the manufacturer's instructions. Lentiviral production was checked with Lenti-X ${ }^{\mathrm{TM}}$ GoStix $^{\mathrm{TM}}$ (Takara Bio, Inc.) and the titer was determined by Enzyme-Linked ImmunoSorbent Assays (ELISA) with the Lenti-X ${ }^{\mathrm{TM}}$ p24 Rapid Titer Kit (Takara Bio, Inc.), according to the supplier's instructions. Then, $4 \mathrm{x}$ $10^{5}$ cells were transduced with at least $5 \times 10^{5}$ IFU viral particles. Positive selection was performed by adding G418 to final concentration of $500 \mu \mathrm{g} / \mathrm{ml}$ (Takara Bio, Inc.) or Puromycin to a final concentration of $1 \mu \mathrm{g} / \mathrm{ml}$ (Sigma-Aldrich Quimica S. de R.L. de C.V.).

\section{Clonogenicity assays and colony formation}

Cell culture plates (p60) were seeded with $5 \times 10^{4}$ cells in $3 \mathrm{ml}$ of medium; after $24 \mathrm{~h}$ of culture, the plates were infected with $2 \times 10^{\text {s }}$ Inclusion-Forming Unit (IFU) of supernatant containing viral particles (LVXPuro-HOXA9 and LVX-Puro empty virus); as control, one plate remained uninfected. At 48-h post-infection, Puromycin was added to cultures at a final concentration of $1 \mu \mathrm{g} / \mathrm{ml}$. Five days later, the cells were fixed with $3.7 \%$ paraformaldehyde, stained with $0.05 \%$ crystal violet, and photo documented. Assays were performed in triplicate. This infection scheme was also evaluated by using the xCELLigence system (Roche Applied Science).

\section{Cell proliferation and viability assays}

The cell proliferation of HOXA9-expressing HeLa cells (in the presence of Doxycycline) was assessed with the xCELLigence system platform (Roche Applied Science). Briefly, $2.5 \times 10^{3}$ cells per well were seeded on a 96-plate E-Plate wells and introduced into the xCELLigence reading station. After $24 \mathrm{~h}$, Doxycycline was added to the culture cells to induce $H O X A 9$ expression; proliferation was assessed in real time every $30 \mathrm{~min}$ during $72 \mathrm{~h}$. Three independent experiments were performed with four duplicates in each case. Cell viability was analyzed by the cleavage of tetrazolium salt Water Soluble Tetrazolium Salts (WST-1) to formazan (Roche Applied Science) by reading the absorbance at $440 \mathrm{~nm}$ on a microtiter plate reader (Synergy ${ }^{\mathrm{TM}}$ HT Multi-Mode Microplate Reader; Biotek Instruments, Inc., Winooski, VT, USA). The value of HOXA9 uninfected cells (HeLa TET) was employed as $100 \%$ cell survival.

\section{Migration assays}

Hela-LVX and HeLa-LVX-HOXA9 cells were seeded onto a p60 plate ( $5 \times 10^{4}$ cells in $3 \mathrm{ml}$ of medium); after 24 $\mathrm{h}$ of culture, the surface of each plate was gently scratched with a cell scraper; cells were washed with PBS, and $3 \mathrm{ml}$ of fresh DMEM medium was added. Photographs were taken at 0,24 , and $48 \mathrm{~h}$ to evaluate the scratches. The photographs were analyzed with ImageJ ver. 1.49 software and the Wound Healing Tool plug-in, which evaluates the wound area (implying the area not covered by cells). The data obtained were graphed employing Microsoft Excel.

\section{Apoptosis detection}

Apoptosis detection was performed by Flow Cytometry (FC) using Annexin-V-FLUOS/Propidium iodide (Roche Applied Science) as markers of cell death. In brief, cells were treated with cold PBS/EDTA and harvested by scraping, packed by centrifugation and washed twice with PBS. Apoptotic cells were incubated for $15 \mathrm{~min}$ at room temperature with Annexin-V-FLUOS in combination with Propidium Iodide (PI). The percentage of positive cells was determined by FC using an Attune Cytometer (Thermo Fisher Scientific, Inc). 
Silencing of E6 and E7 from HPV18 in HeLa cells

The following sequences were employed to silence HPV 18 E6 and E7: GAT CCG CTA ACA CTG GGT TAT ACA ATT CAA GAG ATT GTA TAA CCC AGT GTT AGT TTT TTG (E6/E7 1), and AAT TCA AAA AAC ATT TAC CAG CCC GAC GAG TCT CTT GAA CTC GTC GGG CTG GTA AAT GCG (E6/E7 2); these were previously described elsewhere (Qi et al., 2010). These sequences were cloned into the pLVX-shRNA1 vector (Cat. \# 63217; Takara Bio, Inc.) transfected in HEK cells to produce lentiviruses. The shE6/E7-1 and shE6/E7-2 lentiviruses were utilized to transduce HeLa cells. After infection, cells were selected by adding Puromycin to the cell culture medium.

\section{Statistical analysis}

All experiments were performed in duplicate and repeated independently at least twice. The results are expressed as means \pm Standard Deviation (SD). For differences between groups, analysis was performed using an unpaired, two-tailed Student $\mathrm{t}$-test and statistical significance at set at $\mathrm{p}<0.05$.

\section{Results}

Altered HOX Gene Expression Between Non-tumorigenic Keratinocytes (HaCaT) and CC-derived Cells (HeLa)

With the aim of determining $H O X$ genes differentially expressed in CC-derived cells, a genetic expression comparison between HeLa (CC-derived cell line) and $\mathrm{HaCaT}$ (non-tumorigenic keratinocyte derived cell line) using a microarray-based platform (see Materials and Methods) was performed. Variations in the expression levels of $H O X$ genes were calculated taking the corresponding expression in $\mathrm{HaCaT}$ cells as reference and applying a Student $t$-test to discriminate differences in expression with a $\mathrm{p}$ value of $<0.05$. From this analysis, it was observed that of the 39 human HOX genes, the expression of 26 members remain unchanged in both cell lines; however HOXA3, HOXA9, HOXB4, HOXC12, HOXC13, HOXD10, and HOXD11 were observed with lower expression in HeLa cells compared with HaCaT, and for HOXA11, HOXB3, HOXB5, HOXB6, HOXB9, and $H O X B 13$, increased expression was observed (Figure 1a). To validate the observations of the microarray experiments, differentially expressed genes were evaluated by quantitative real time PCR in HaCaT and HeLa cells. For these assays, three reference genes (GAPDH, PGK1, and RPLP0) were used for normalization, and expression of $\mathrm{HaCaT}$ cells was utilized as calibrator (set as 1). With the exception of HOXD11, the expression of all analyzed genes was confirmed by qPCR (Figure 1b). The downmodulation of $H O X A 9$ is noteworthy, with a relative expression value of 0.09 . This gene is involved in the regulation of endothelial cell proliferation and has been related with a tumorigenic suppression in some epithelial models. In order to evaluate the possible participation of HOXA9 in cervical carcinogenesis, we analyzed this gene further.
HOXA9 mRNA Expression Is Diminished in CC Cell Lines

With the purpose of knowing whether downregulation of HOXA9 is a common characteristic of CC cells the expression of this gene was analyzed by qPCR in additional $\mathrm{CC}$-derived cell lines: $\mathrm{SiHa}$ (positive to HPV16), and C-33A (negative to HPV infection). Because SYBR green-based detection was used for the qPCR, the melting curves of the amplicons were determined to discard unspecific amplifications, and the expected Molecular Weights (MW) of qPCR products were also confirmed on agarose gels (Figure $2 \mathrm{a}$ ). The relative expression of $\mathrm{HOXA} 9$ was determined in $\mathrm{HeLa}$, $\mathrm{SiHa}$, and $\mathrm{C}-33 \mathrm{~A}$ cells using the expression of $\mathrm{HaCaT}$ as calibrator. A significant decrease in $H O X A 9$ levels was observed in the three tumorigenic cell lines, with C-33A the cell line showing lowest expression ( 0.0099 -fold), followed by $\mathrm{HeLa}$ (0.06-fold), and SiHa (0.38-fold) (Figure 2a).

CC biopsies showed lower expression of HOXA9 mRNA than control cervical cells

To determine whether the diminished expression of HOXA9 observed in CC-derived cell lines is also present in CC samples, nine samples of biopsies from patients with $\mathrm{CC}$ and 18 cervical scrapes from women without obvious cervical lesions were recruited (Figure 2b). HOXA9 mRNA levels were analyzed and expression values were calculated for each patient by $\Delta \mathrm{Cp}$ analysis, that is, the $\mathrm{Cp}$ of the HOXA9 gene minus the expression of a reference gene from the same patient; from this analysis, it was observed that controls have lower $\Delta \mathrm{Cp}$ values, indicating

a)
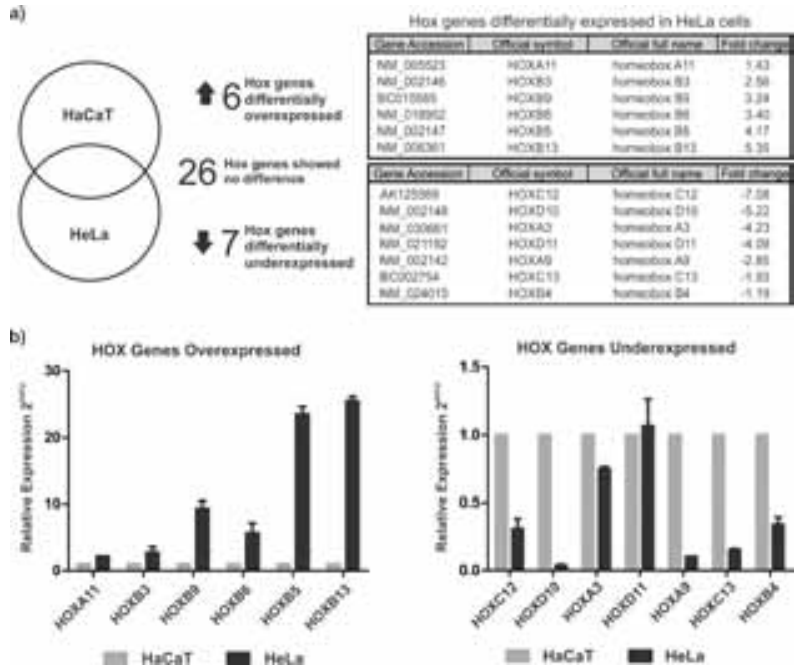

Figure 1. Messenger RNA (mRNA) Expression of HOX Genes in HeLa vs. HaCaT Cells. (a) Venn diagram of differentially expressed genes in HeLa when compared with $\mathrm{HaCaT}$ cells determined by microarray analysis (statistically significant, $\mathrm{p} \leq 0.005$ ). In the right panel, overexpressed or underexpressed HOX genes in HeLa cells are shown, including gene accession number, official symbol, official full name, and fold -change. (b) Validation using qPCR of over- and underexpressed genes selected from microarray expression assays; relative expression is shown as fold change $(2 \Delta \Delta \mathrm{Cp})$. Values obtained were normalized to three reference genes: GAPDH; PGK1, and RPLP0, and HaCaT cells were employed as calibrator (set as 1 ). Means \pm SD from at least three independent experiments are shown 

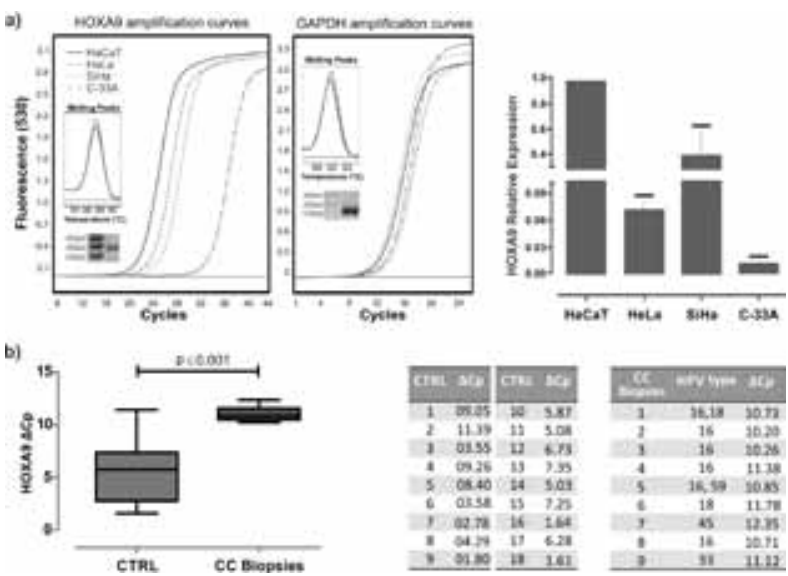

Figure 2.HOXA9 Expression in Normal Cervical Cell vs. CC-derived Cells. (a) In the left panel, representative graphics of the amplification curves obtained from HOXA9 and the reference gene GAPDH are depicted for HaCaT, HeLa, $\mathrm{SiHa}$, and C-33A cell lines. Agarose gels of PCR products and melting peaks are also depicted. Right panel shows a graphic of HOXA9 relative expression $(2 \Delta \Delta \mathrm{Cp})$; the values depicted were normalized to three reference genes (GAPDH, ACTB, and PGK1) and were determined relative to HaCaT. (b) Relative HOXA9 mRNA expression depicted as $\triangle \mathrm{Cp}$ in cervical scrapes of healthy women (CTRL) and cervical biopsies with Cervical Cancer (CC Biopsies). $\triangle \mathrm{Cp}$ was calculated by subtracting the $\mathrm{Cp}$ value of the reference gene (GAPDH) of one sample from the Cp obtained for the HOXA9 gene of the same sample. The graph depicts medians (dark lines), 5-95 percentile limits (boxes), and InterQuartile Ranges (IQR) (Whiskers). Two-tailed Student $t$ -test $(p<0.001)$. Tables right. Information on the samples of controls: sample number and $\Delta \mathrm{Cp}$ obtained in the analysis. Information of patients with CC biopsies: patient number, HPV genotype positivity, and $\Delta \mathrm{Cp}$

that HOXA9 expression is higher in controls than in patients with CC. These differences were statistically significant and correlated with our previous results.

The HOXA9 Promotor Is Methylated in CC Cell Lines, But Not in Keratinocytes and HaCaT Cells

To determine whether the expression of HOXA9 is reduced in tumor cells due to hypermethylation, as was previously described in other tissues, the methylation status of five different zones belonging to four $\mathrm{CpG}$ islands located within the promoter and gene sequence of HOXA9 $(-1,400 \mathrm{pb}$ to $+800 \mathrm{pb})$ was evaluated by restriction with methylation-sensitive and -insensitive restriction enzymes (HpaII and MspI, respectively), followed by PCR (Figure 3a, see Materials and Methods). As depicted in Figure 3b, $\mathrm{HaCaT}$ cells demonstrated the methylation of Zones 1 and 5 , localized at the ends of the analyzed area, but Zones 2, 3, and 4 , which correspond to the near promoter region and to the first exon, were not methylated. In contrast, $\mathrm{HeLa}$ and C-33A cells exhibited methylation in all analyzed zones (Z1-Z5). Interestingly, SiHa cells have a pattern similar to that of $\mathrm{HaCaT}$ cells (Z1- and Z5-methylated) with some methylation in $\mathrm{Z} 2$, which corresponds to the promoter region. As control, the methylation status of normal cervical primary keratinocytes isolated from a patient without cervical lesions and without HPV infection, was also included; normal keratinocytes demonstrated nearly
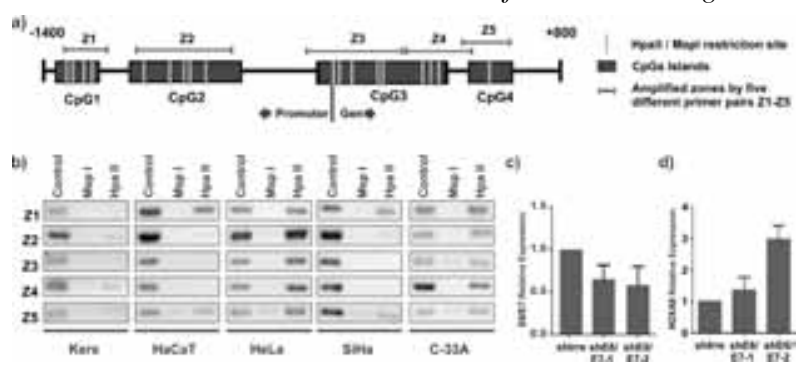

Figure 3. Methylation Status of HOXA9 and HOXA9 mRNA Regulation by HPV18 E6/E7 Oncogenes. (a) Representative image of the sequence: $-1,400 \mathrm{bp}$ to $+800 \mathrm{bp}$ from the transcription start of the HOXA9 gene, predicted $\mathrm{CpG}$ islands, PCR-amplified regions (Z1-Z5), and restriction sites (MspI/ HpaII) are depicted as dark boxes, horizontal lines, or vertical light-grey lines, respectively. (b) Gel electrophoresis of the amplification products for Z1-Z5 after restriction with MspI or HpaII for HaCaT, HeLa, SiHa, and C-33A cell lines and normal primary Keratinocytes (Kers). (c) Relative HOXA9 expression in HeLa cells transduced with shRNA coding virus directed to E6/E7 of HPV18 (shE6/E7-1 and shE6/E7-2), and irrelevant shRNA was also used as control (shIrre). Expression values of E6/E7 and HOXA9 were calculated relative to the expression observed in HeLa-shIrre. For normalization, reference genes RPL32, RP18S, and RPLP0 were used

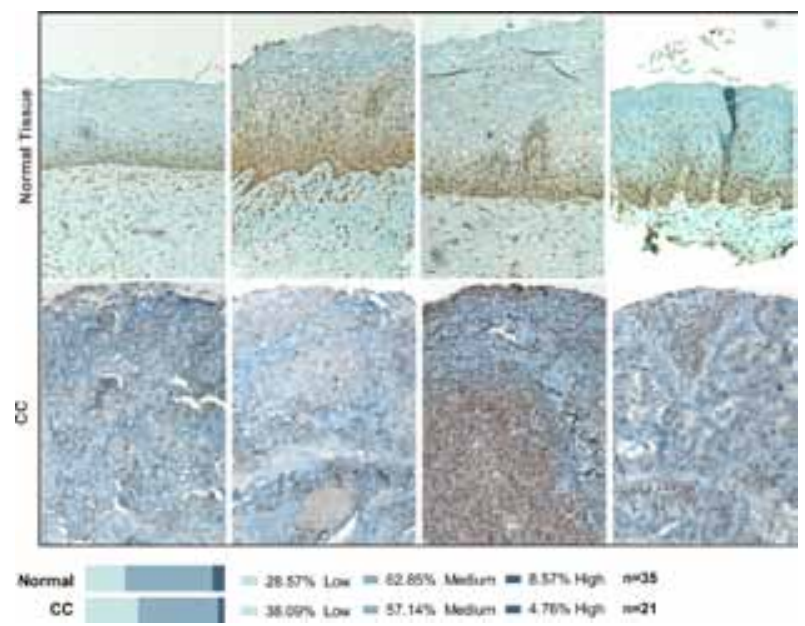

Figure 4. Immunodetection of $\mathrm{HOXA9}$ in Normal Cervical and Cervical Cancer (CC) tissues. $\mathrm{CC}$ biopsies and normal cervical tissues were used to detect HOXA9 by immunohistochemistry with an anti-HOXA9 antibody. Representative images are presented at a magnification of 10X. HOXA9 expression was evaluated and classified according to degree of positivity: negative; low; medium, or high, and the percentages are included at the bottom of the image

no methylation in all analyzed zones.

\section{HPV18 E6 and E7 Oncoproteins Regulates HOXA9 Expression}

Our previous results indicated that HOXA9 was methylated in some CC-derived cell lines, particularly in the HPV18-infected HeLa cell line. To address the possible participation of HPV 18 oncoproteins in methylation status, shRNA designed to target the E6 and E7 of HPV18 were transduced in HeLa cells (see Material and Methods). After this, expression of E6 and E7 at the mRNA level 
Supplementary Table 1. Primer pairs utilized for PCR and qPCR

\begin{tabular}{|c|c|c|c|}
\hline GENE & GenBank ID & Sequence & Size \\
\hline HOXA9 & NM_152739.3 & $\begin{array}{l}\text { F- TTGTCCCTGACTGACTTGCT } \\
\text { R-GTCCCTGGTGAGGTACATGTT }\end{array}$ & $249 \mathrm{pb}$ \\
\hline ACTB & NM_001101.3 & $\begin{array}{l}\text { F- CGGCTTCCTTTGTCCCCAATC } \\
\text { R-CAGAGAAAGCGCCCTTGCCT }\end{array}$ & $316 \mathrm{pb}$ \\
\hline GAPDH & NM_002046 & $\begin{array}{l}\text { F- CACTGCCACCCAGAAGACTGTG } \\
\text { R-TGTAGGCCATGAGGTCCACCAC }\end{array}$ & $449 \mathrm{pb}$ \\
\hline RPS18 & NM_022551.2 & $\begin{array}{l}\text { F-CGATGGGCGGCGGAAAA } \\
\text { R-CAGTCGCTCCAGGTCTTCACGG }\end{array}$ & $283 \mathrm{pb}$ \\
\hline RPLP0 & NM_001002.3 & $\begin{array}{l}\text { F- CCTCATATCCGGGGGAATGTG } \\
\text { R- GCAGCAGCTGGCACCTTATTG }\end{array}$ & $95 \mathrm{pb}$ \\
\hline HOXA9 ORF & NM_152739.3 & $\begin{array}{l}\text { F- CAGTTTCATAATTTCCGTGGGTC } \\
\text { R- CTGAGGTTTAGAGCCGCTTTGT }\end{array}$ & $1011 \mathrm{pb}$ \\
\hline HOXA11 & NM_005523.5 & $\begin{array}{l}\text { F- CTTCCGGCCACACTGAGGACA } \\
\text { R-GCCGCTGGAGTCTTAGAGGAG }\end{array}$ & $281 \mathrm{pb}$ \\
\hline HOXB3 & NM_002146.4 & $\begin{array}{l}\text { F- CCGGCACCAACTCCACCCTCA } \\
\text { R-TCTTGATCTGCCGCTCGCTGA }\end{array}$ & $445 \mathrm{pb}$ \\
\hline HOXB4 & NM_024015.4 & $\begin{array}{l}\text { F- CGTCCCACTCCGCGTGCAAA } \\
\text { R-TCCACTTCATGCGCCGGTTCT }\end{array}$ & $261 \mathrm{pb}$ \\
\hline HOXB5 & NM_002147.3 & $\begin{array}{l}\text { F- CGCGTCCTCGGAGCCTGAG } \\
\text { R-AGCGGTTGAAGTGGAACTCCT }\end{array}$ & $242 \mathrm{pb}$ \\
\hline HOXB6 & XM_005257284. 2 & $\begin{array}{l}\text { F-CGTGTTCGGCGAGACAGAAGA } \\
\text { R-TGAGACGCGCTGAGCAGTTT }\end{array}$ & $297 \mathrm{pb}$ \\
\hline HOXB9 & NM_024017.4 & $\begin{array}{l}\text { F-CGCCGTGCTGTCTAATCAAAG } \\
\text { R-TTGCCCTGCTCCTTATTCATT }\end{array}$ & $331 \mathrm{pb}$ \\
\hline HOXB13 & NM_006361.5 & $\begin{array}{l}\text { F-AACCGCGACATGACTCCCTGT } \\
\text { R-GGCGCTCCGAGAGGCTGGTG }\end{array}$ & $306 \mathrm{pb}$ \\
\hline $\mathrm{HOXC} 12$ & NM_173860.1 & $\begin{array}{l}\text { F-ACGACCCGCCCTCCTGCCAGT } \\
\text { R-AACTCGCCCTCCAGCTCTGCC }\end{array}$ & $226 \mathrm{pb}$ \\
\hline $\mathrm{HOXC13}$ & NM_017410.2 & $\begin{array}{l}\text { F-TGCCCGGCTACCTGGACGTGT } \\
\text { R-TGAACTTGCTAGCCGCGTATT }\end{array}$ & $300 \mathrm{pb}$ \\
\hline HOXD10 & NM_002148.3 & $\begin{array}{l}\text { F-CTCCGTGTCCAGTCCCGAAGT } \\
\text { R-AGGTTGGCGGTCAGTTCTC }\end{array}$ & $306 \mathrm{pb}$ \\
\hline HOXD11 & NM_021192.2 & $\begin{array}{l}\text { F-GGGGGCCGAGAAGAGCAGCAG } \\
\text { R-GGGTGAGGGCGCTTCCTGGAG }\end{array}$ & $286 \mathrm{pb}$ \\
\hline $\mathrm{CDH} 1$ & NM_001317184.1 & $\begin{array}{l}\text { F-CCTCATGAGTGTCCCCCGGTAT } \\
\text { R- CTCGCCGCCTCCGTACATGTC }\end{array}$ & $271 \mathrm{pb}$ \\
\hline GRHL2 & NM_024915.3 & $\begin{array}{l}\text { F-GAAGAAGGGACAAAGCGAGTG } \\
\text { R-CCAAACCCAGGGCTAGATTTC }\end{array}$ & $297 \mathrm{pb}$ \\
\hline TCF4 & NM_001083962.1 & $\begin{array}{l}\text { F-TCGGAGGACAAGAAATTAGAT } \\
\text { R-CGCAGCTTTCGGATTC }\end{array}$ & $321 \mathrm{pb}$ \\
\hline TWIST1 & NM_000474.3 & $\begin{array}{l}\text { F-CGCGTCCCACTAGCAG } \\
\text { R-TGGCTGATTGGCACGACCTCT }\end{array}$ & $393 \mathrm{pb}$ \\
\hline WNT5A & NM_003392.4 & $\begin{array}{l}\text { F-TTGGTGGTCGCTAGGTATGAAT } \\
\text { R-ACCTTCGATGTCGGAATTGATA }\end{array}$ & $203 \mathrm{pb}$ \\
\hline WNT5B & NM_032642.2 & $\begin{array}{l}\text { F-GGGAGCGAGAGAGAAGAACTTTGC } \\
\text { R-GGGCTGGGGTCCACATAGAC }\end{array}$ & $319 \mathrm{pb}$ \\
\hline
\end{tabular}

Supplementary Table 2. Primer pairs utilized for amplification of HOXA9 promotor CpG islands

\begin{tabular}{|cccc|}
\hline & POSITION & SEQUENCE & SIZE \\
\hline Z1 & $1655-1906$ & F- CCTTGTACCTCTCCGCCGAGTC & $251 \mathrm{pb}$ \\
& & R- CTGGTTGCGCTCCGTGAATC & $514 \mathrm{pb}$ \\
$\mathrm{Z2}$ & $2051-2369$ & F-GCCTGCGCCTGGGAATCCTGA & $455 \mathrm{pb}$ \\
& & R- GGCTGGCGGGAAGTCGGAAAC & $386 \mathrm{pb}$ \\
$\mathrm{Z3}$ & $2951-3202$ & F- GCGGCACGATCC CTTTACATA & $298 \mathrm{pb}$ \\
& & R-CACCGTCGCCTTGGACTGGAA & \\
& $3376-3486$ & F-CTTCCAGTCCAAGGCGACGGT & R-TCTCCGCCGCTCTCATTC T \\
\end{tabular}


HOXA9 is Underexpressed in Cervical Cancer Cells and its Restoration Decreases Proliferation and Migration
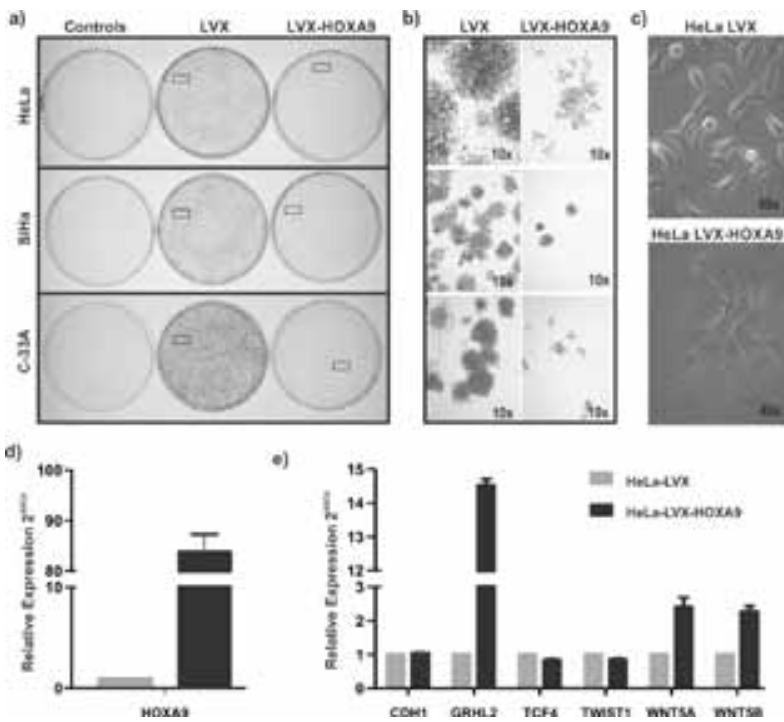

Figure 5. Effects on Clonogenic and Phenotype of CCDerived Cells After Restoration of $\mathrm{HOXA9}$ Expression. (a) Clonogenic cell-survival assays performed in $\mathrm{HeLa}, \mathrm{SiHa}$, and C-33A cell lines. Cells lines were transduced with Lentivirus carrying a Puromycin-resistant gene (LVX) and Puromycinresistant plus HOXA9 gene (LVX-HOXA9); non-transduced cells were included as controls. After 6 days of Puromycin treatment, cells were fixed and colonies were evaluated. (b) $10 \mathrm{X}$ magnification of colonies. (c) $40 \mathrm{X}$ magnification of nontransduced and HOXA9-transduced HeLa cells. (d) Relative expression of HOXA9 in HeLa cells transduced with LVX and LVX-HOXA9. (e) Gene expression evaluation of epithelialto-mesenchymal transition markers in HeLa transduced with HOXA9. Relative expression was calculated as related with the expression of HeLa transduced with the empty vector (HeLaLVX). Values were normalized using GAPDH and ACTB as reference genes

was measured by qPCR. Because two different shRNA directed to E6 and E7 genes were used, the silenced HeLa cells were denominated shE6/E7-1 and shE6/E72. As illustrated in Fig 3c, expression of the oncogenes diminished from 1- to 0.63 -fold for shE6/E7-1 and from 1- to 0.58 -fold for shE6/E7-2. Utilizing these models, the HOXA9 expression was also evaluated by qPCR. As can be observed in Figure 3d, HOXA9 expression increases in both models of E6/E7 silenced cells, indicating possible participation of HPV oncogenes in the regulation of HOXA9.

HOXA9 Protein Expression Was Diminished in CC Tissues

To corroborate these previous observations, HOXA9 expression was evaluated by immunohistochemistry in biopsies from patients with $\mathrm{CC}$, and also in healthy cervical samples as control. The HOXA9 signal was evaluated by a Pathologist and classified as negative, low (+), medium (++), and high (+++), according to the positivity. The percentages of different degrees of HOXA9 expression were calculated and represented graphically (Figure 4). It was found that $8.57 \%$ of normal cervicaltissue samples have high $H O X A 9$ expression, $62.8 \%$ medium expression, and $28.57 \%$ low expression; instead, in $\mathrm{CC}$ samples, high $\mathrm{HOX} A 9$ expression decreases to $4.76 \%$, medium expression decreases to $57.14 \%$, and low
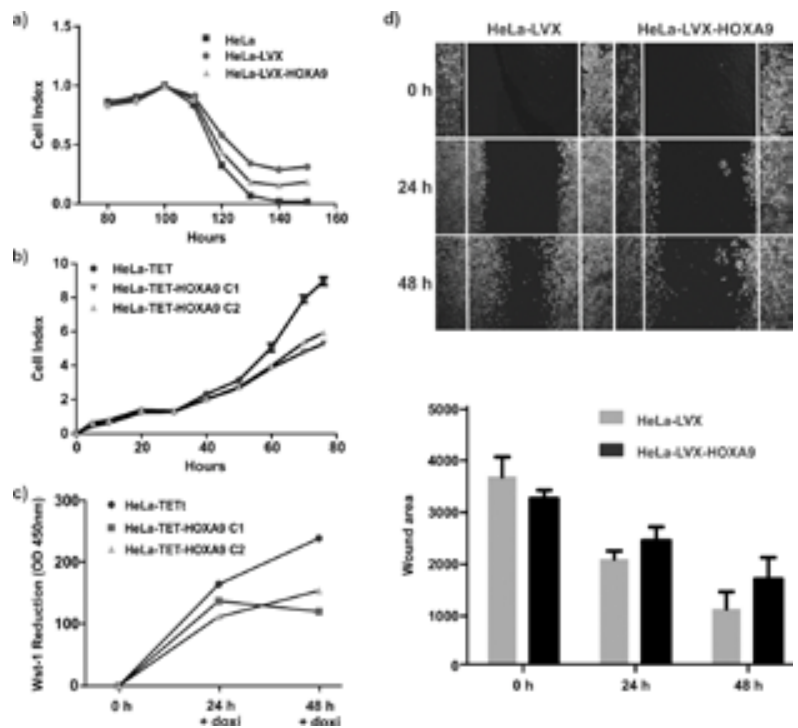

Figure 6. Effect of $\mathrm{HOXA9}$ Restoration in Survival, Proliferation, Metabolism, and Migration. (a) Rate of survival (measured as cell index) after Puromycin treatment. HeLa cells were infected ( $24 \mathrm{~h}$ after seeded onto an E-Plate) with Lentivirus carrying a Puromycin-resistant gene (HeLaLVX) and a Puromycin-resistant plus HOXA9 gene (HeLa LVX-HOXA9); non-transduced cells were included as controls. After $72 \mathrm{~h}$, Puromycin was added and cell density was recorded every $30 \mathrm{~min}$. (b) Proliferation rate (measured as cell index). HOXA9 expression was induced by Doxycycline treatment, and its growth was evaluated in HeLa-TET-HOXA9 (two different clones) cells. HeLa-TET cells were also included as controls. (c) Metabolism rate measured as reduction of WST-1 at $24 \mathrm{~h}$ and at $48 \mathrm{~h}$. Expression of HOXA9 was induced by Doxycycline treatment in the previously described cell lines (panel b). (d) Scratch assays were performed to evaluated migration of HeLa-LVX and HeLa-LVX-HOXA9 cells at $24 \mathrm{~h}$ and at $48 \mathrm{~h}$. Representative photographs are presented in the upper panels, and the uncover area was calculated, and graphically shown in the lower panel

expression increases to $38.09 \%$. These results indicate that protein expression of HOXA9 has a tendency to decrease in $\mathrm{CC}$ when compared with normal cervical tissues.

Exogenous HOXA9 Expression Induced Lower Colony Formation in CC-derived Cell Lines and Modified the Cellular Phenotype

The questions that arose from the previous observations including the following: why was the expression of HOXA9 diminished in CC-derived cell lines and in CC biopsies, and was diminution of HOXA9 an advantage to cancer-cell establishment. To determine whether the presence of HOXA9 is compatible with cell growth and proliferation, expression of HOXA9 was exogenously restored in CC-derived cells. For this, lentiviral particles carrying a Puromycin-resistant gene (LVX) and the Puromycin-resistant plus HOXA9 reading frame (LVXHOXA9) were produced; thus, only infected cells would be able to survive and form colonies when cultured in the presence of Puromycin. As observed in Figure 5a, cells that were not infected did not survive and no colony formation was observed; instead, cells infected with viral particles carrying only the Puromycin-resistant gene (LVX) formed 
a great number of colonies. However, cells infected with viral particles carrying the Puromycin-resistant gene and HOXA9 (LVX-HOXA9) formed low numbers of colonies (Figure 5b), indicating that the presence of HOXA9 affects the growth of CC-derived cells.

In addition, it was observed that infection with exogenous HOXA9 not only decreased the number of colonies formed, but also changed cellular morphology, that is, HeLa cells, which normally grow isolated with no visible cell junctions and with mesenchymal morphology, in presence of HOXA9 cells have larger and irregular borders with indicia of cell junctions and epithelial morphology (Figure 5c). Due to the observation of such changes, we decided to evaluate modifications in the expression of genes involved in the epithelialto-mesenchymal transition (CDH1, GRHL2, TCF4, TWIST1, WNT5A, and WNT5B) after restoration of HOXA9 (Figure 5d). We found that genes such as GRHL2, which is normally present in epithelial, and not in mesenchymal cells, increase their expression when HOXA9 is restored. Likewise, the TCF4 and TWIST1 genes, which are related with the mesenchymal phenotype, decrease their expression 0.75 -fold and 0.79 -fold, respectively (Figure 5e).

Restoration of HOXA9 Expression Decreases Cell Proliferation and Cell Metabolism, But Does Not Induce Apoptosis in CC-derived Cell Lines.

The results of clonogenic assays suggest a modulation of cell proliferation and viability by HOXA9. This hypothesis was further evaluated by measuring the cell growth of HeLa cells infected with viral particles carrying the Puromycin-resistant gene alone or with HOXA9. Cell growth was evaluated in real time using the Real-Time Cell Analyzer (RTCA) xCELLigence System. Cells were seeded, infected with viral particles and, after 72 $\mathrm{h}$, Puromycin was added to the culture. As presented in Figure 6a, uninfected HeLa cells were not able to survive and died at $120 \mathrm{~h}$ of culture. Cells infected with lentiviral particles carrying the Puromycin-resistant gene only (LVX) exhibited growth curves that indicated more surviving cells; in turn, cells infected with the Puromycinresistant gene and HOXA9 (LVX-HOXA9) displayed growth curves indicating a lower number of surviving cells in comparison with those shown with LVX alone. These results corroborate the negative effect that HOXA9 exert on the growth of the CC-derived cell line. Employing another strategy, a conditional model (TETracyclinecontrolled Transcriptional Activation [TET] system) was additionally developed, in which HOXA9 expression is controlled by the presence of Doxycycline in the culture; two different clones were obtained. Using this system, cell proliferation was also evaluated with and without the inducer of $H O X A 9$ expression. As displayed in Figure 6b, induction of HOXA9 reduced cell proliferation in both clones.

Taking advantage of the previously developed conditional model, cell metabolism was also evaluated by using WST-1. As depicted in Figure $6 \mathrm{c}$, we found that HOXA9 expression strongly decreases cell metabolism; similar results were also obtained using the SiHa cell line (data not shown).

Exogenous HOXA9 Expression Decreases the Migration Rate of HeLa Cells

Another feature of cancer cells is an increased ability to migrate. In order to test whether exogenous HOXA9 expression modulated CC-cell migration, scratch assays were performed on cultures of HeLa cells infected with LVX-Puro (viral particles only with the Puromycin gene) and with LVX-HOXA9 cells (viral particles with the HOXA9 and the Puromycin gene). Time for woundclosure was evaluated at $0 \mathrm{~h}, 24 \mathrm{~h}$, and $48 \mathrm{~h}$. As can be observed in Figure 6d, cells expressing HOXA9 migrate more slowly than cells without it. Images of cell growth were analyzed by the "ImageJ" computer program to calculate the uncovered area of the wound. These analyses showed that, although initially $(0 \mathrm{~h})$, the wound area of HeLa-LVX-Puro cells was slightly larger, it decreased faster than the wound area of HeLa-LVX-HOXA9.

Because HOXA9 decreases colony formation, cell proliferation, cell migration, and cell metabolism, it was in our interest to evaluate whether HOXA9 also modulates the apoptosis process. The percentage of apoptotic cells in the presence or not of HOXA9 was evaluated and no differences between early and late apoptosis, comparing Hela-LVX vs. Hela-LVX-HOXA9 and HeLa-TET vs. HeLa-TET-HOXA9 were observed (data not shown).

\section{Discussion}

$H O X$ genes encode transcription factors that regulate and determine different cell types during embryonic development (Castelli-Gair, 1998). They are considered as master regulators involved in fundamental processes during the development of an organism (Svingen and Tonissen, 2006). In recent years, abnormal expression of these genes has been linked with the emergence of various, solid and hematopoietic malignancies (16-20). In $\mathrm{CC}$, there are evidences concerning the overexpression or downregulation of $H O X$ members, indicating its possible role during cervical carcinogenesis (Lopez et al., 2006b; Gonzalez-Herrera et al., 2015). In order to underscore differences in $H O X$ genes expression in $\mathrm{CC}$ and to corroborate and complement previous findings, we performed a comparison by microarray of the mRNA expression profile of two cell lines: one derived from CC (HeLa), and the other, from non-tumorigenic keratinocytes (HaCaT). A differential expression of HOXA3, HOXA9, and $H O X A 11$ genes in HeLa compared with HaCaT cells was found. The HOXA cluster has been related with normal development of urogenital tracts (Burel et al., 2006); in particular, HOXA9 and -11 are important in controlling the embryonic development of Müller ducts (Hsieh-Li et al., 1995). With regard to HOXA9, this gene has been found overexpressed in hematological malignancies (Milne et al., 2010)\{Zhao, 2014 \#203\}, but in solid tumors, especially epithelial tumors, it has been observed as downregulated (Gilbert et al., 2010; Chen et al., 2012; Hwang et al., 2015). These observations were in agreement with our observations. We found that HOXA11 was slightly upregulated in Hela cells compared with 
HaCaT. The expression of HOXA11 was found unchanged in normal and malignant cervical cells (Lopez et al., 2006b). A report from Bai et al. indicates that HOXA11 is regulated by methylation and that the expression of this gene decreases in gastric cancer; moreover, these authors observed that silencing of HOXA11 induced cell proliferation of tumor cells (Bai et al., 2014).

Concerning the $H O X$ B locus, we observed a modulation of $H O X \mathrm{~B} 3,-4,-5,-6,-9$, and -13 , and of these, only $H O X \mathrm{~B} 4$ was found to be downregulated, while the remaining genes were overexpressed. According to our results, overexpression of $H O X \mathrm{~B}$ genes has been observed in CC (Lopez et al., 2006a). Lopez et al. found the expression of $H O X \mathrm{~B} 1,-\mathrm{B} 3,-\mathrm{B} 5,-\mathrm{B} 6,-\mathrm{B} 7,-\mathrm{B} 8$, and -B9 genes in normal cervix epithelia and in SCC. Moreover, the authors found the expression of $H O X \mathrm{~B} 2$, -B4, and -B13 only in tumor tissues. We did not find variations in the expression of $H O X \mathrm{~B} 2$ and observed downregulation of $H O X \mathrm{~B} 4$, perhaps due to the different approach and the targets measured (we measured mRNA and Lopez et al., protein). Despite these dissimilarities, we also found upregulation of $H O X \mathrm{~B} 13$. This phenomenon has also been observed in other works (Lopez et al., 2006b; GonzalezHerrera et al., 2015) \{Gonzalez-Herrera, 2014 \#204\}, and in other cancer types (Zhu et al., 2014; Barresi et al., 2015)\{Long, 2013 \#205\}\{Zhu, 2011 \#206\}. Regarding the $H O X C$ cluster, we observed reduced expression of HOXC12 and -C13 in the CC-derived cell line HeLa. HOXC12 was recently proposed as a tumor suppressor gene downregulated by methylation in head and neck SCC (Guerrero-Preston et al., 2014). HOXC13 regulates the expression of keratins, and abnormal expression of this gene has been observed in malignant pilomatricoma (Jave-Suarez et al., 2002; Cribier et al., 2006); in SCC, expression of HOXC13 is augmented and modulated by histone methylation (Marcinkiewicz and Gudas, 2014b; Marcinkiewicz and Gudas, 2014a). Overexpression of $H O X C 13$ was additionally observed in melanoma (Cantile et al., 2012), and functional studies indicate that overexpression of $\mathrm{HOXC} 13$ induces proliferation and tumorigenesis; conversely, silencing of this gene induces apoptosis and cell cycle arrest (Kasiri et al., 2013).

HOXD10 was observed with lower expression in HeLa cells compared with $\mathrm{HaCaT}$, and the expression of HOXD11 remains unchanged in both cell lines. Posterior HOXD and -A (9-11) genes have redundant functions in reproductive organ development, and mutations in these genes induce changes in the epithelia, from columnar to squamous, and promote the migration of myeloid suppressor cells (Raines et al., 2013). In this regard, downmodulation of HOXD10 and HOXA9 could be involved in the maintenance of the epithelial phenotype and in the modulation of the immune response. The discrepancies between our data and those previously reported in relation to $H O X \mathrm{~B} 4, H O X \mathrm{C} 12$, and $H O X \mathrm{C} 13$ may be due to the different techniques used, as well as to the new isoforms recently described (Brunskill and Potter, 2012).

Particularly with HOXA9, we observed that the proximal sequence ( -200 bases) at the transcription start and the following $(+150)$ bases after this, comprises an area of critical importance for the regulation of HOXA9 mRNA, and that it is a principal area of methylation. Our observations are in agreement with those shown in other models of epithelial malignancies; methylation and HOXA9 loss of expression have been reported in breast, ovarian, bladder, oral, and lung cancer (Rauch et al., 2007; Wu et al., 2007; Guerrero-Preston et al., 2011; Kim et al., 2013; Conway et al., 2014). All of these observations Indicate a possible role of $H O X A 9$ as a tumor suppressor gene in epithelial cells; unlike its well described oncogenic potential in hematopoietic malignancies (Collins and Hess, 2015).

The tumor suppressor function of HOXA9 has been extensively investigated in breast cancer, where it has been shown that HOXA9 directly regulates BRCA1 and other genes involved in invasion, growth, and metastasis (Gilbert et al., 2010). Uchida et al. observed that high methylation levels of HOXA9 are associated with a high risk of metastatic tumors; in addition, the authors found that reduced HOXA9 expression confers an advantage on cell growth (Uchida et al., 2014); these observations are consistent with our results in CC-derived cells. We observed that restoration of HOXA9 expression in CCcells lines infected with HPV 18 significantly decreases cell growth, colony formation, metabolism, and cell migration. Contrary to our results, Sun et al. observed high expression of HOXA9 in laryngeal SCC compared with normal mucosal cells; also, using liver hepatocellular cells, these authors demonstrated that downregulation of HOXA9 inhibits cell growth (Sun et al., 2013). Conversely and in agreement with our results, Uchida et al. observed that re-expression of HOXA9 in oral cancer cell lines inhibits cell growth (Uchida et al., 2014). The different tissues employed in those experiments could explain the controversial observations; hepatocellular cells are different from oral cancer cells, which derive from squamous epithelial tissue, as do CC cells.

We also observed that HOXA9 restoration induces morphological changes that resemble the epithelial phenotype. Because HOXA9 regulates the expression of cadherins (VE-Cadherin and P-Cadherin) (Rossig et al., 2005; Ko and Naora, 2014), we evaluated the expression of E-cadherin (CDH1), together with other important markers of epithelial-to-mesenchymal transition. The expression of CDH1, TCF4, and TWIST1 was slightly modified by the restoration of HOXA9; however, GRHL2 was dramatically overexpressed, i.e, $>14$ times compared with the control. GRHL2 is a novel suppressor of epithelial to mesenchymal transition that is expressed in normal cervical epithelium and with reduced expression in CC cells (Torres-Reyes et al., 2014; Mehrazarin et al., 2015). In agreement to these results, variations in the expression of HOXA9 were previously observed by changes in epithelial and mesenchymal interactions in the intestine, suggesting a role of this gene in epithelial and mesenchymal communication (Duluc et al., 1997).

An important observation was the upregulation of HOXA9 when HPV oncogenes were silenced. There is no previous evidence of $H O X A 9$ regulation by HPV infection, or of $H O X$ genes regulation by the E6 or E7 oncoproteins. More studies are needed in this regard, and our group is 
currently working on this. Although our study reinforces the role of HOXA9 as tumor suppressor in the cervical epithelia, many questions remain concerning $H O X A 9$ that have arisen from our and other observations. It is important to emphasize that the dual behavior of HOXA9 as an oncogene or tumor suppressor highlights the importance of tissue context in its function.

In summary, $\mathrm{HOX}$ 99 expression is downmodulated in some types of CC cells, and that could depend on the HPV type that it infects. HOXA9 downmodulation represents an advantage for HPV18-infected CC cells, which in that condition are more proliferative and have a high migration rate. The expression of $H O X A 9$ is regulated by methylation and by the HPV oncogenes, and the restoration of HOXA9 induces lowers proliferation and the markers of ephitelialto-mesenchymal transition.

Conflict of interest: The authors declare that the research was conducted in the absence of any commercial or financial relationships that could be construed as a potential conflict of interest.

Ethical approval: "All procedures performed in studies involving human participants were in accordance with the ethical standards of the institutional and/or national research committee and with the 1964 Helsinki declaration and its later amendments or comparable ethical standards."

\section{Acknowledgements}

We thank our technician Leticia Ramos-Zavala for her efficient support in the laboratory and Humberto GarciaJiménez for his support with the recruitment of patients. Liliana Alvarado-Ruiz is grateful for scholarships obtained from CONACyT-Mexico. This work was supported by grant FIS/IMSS/PROT/G11-2/1016 grant to LFJS.

\section{References}

Acampora D, D'Esposito M, Faiella A, et al (1989). The human HOX gene family. Nucleic Acids Res, 17, 10385-402.

Alami Y, Castronovo V, Belotti D, et al (1999). HOXC5 and HOXC8 expression are selectively turned on in human cervical cancer cells compared to normal keratinocytes. Biochem Biophys Res Commun, 257, 738-45.

Bai Y, Fang N, Gu T, et al (2014). HOXA11 gene is hypermethylation and aberrant expression in gastric cancer. Cancer Cell Int, 14, 79 .

Barresi V, Ieni A, Cardia R, et al (2015). HOXB13 as an immunohistochemical marker of prostatic origin in metastatic tumors. APMIS.

Bosch FX, Munoz N (2002). The viral etiology of cervical cancer. Virus Res, 89, 183-90.

Brunskill EW, Potter SS (2012). RNA-Seq defines novel genes, RNA processing patterns and enhancer maps for the early stages of nephrogenesis: HOX supergenes. Dev Biol, 368, 4-17.

Burel A, Mouchel T, Odent S, et al (2006). Role of HOXA7 to HOXA13 and PBX1 genes in various forms of MRKH syndrome (congenital absence of uterus and vagina). J Negat Results Biomed, 5, 4.

Cantile M, Scognamiglio G, Anniciello A, et al (2012). Increased HOX $\mathrm{C} 13$ expression in metastatic melanoma progression. $J$ Transl Med, 10, 91.

Castelli-Gair J (1998). Implications of the spatial and temporal regulation of $H O X$ genes on development and evolution. Int J Dev Biol, 42, 437-44.

Collins CT, Hess JL (2015). Role of HOXA9 in leukemia: dysregulation, cofactors and essential targets. Oncogene.

Conway K, Edmiston SN, May R, et al (2014). DNA methylation profiling in the Carolina Breast Cancer Study defines cancer subclasses differing in clinicopathologic characteristics and survival. Breast Cancer Res, 16, 450.

Coutlee F, Rouleau D, Petignat P, et al (2006). Enhanced detection and typing of human papillomavirus (HPV) DNA in anogenital samples with PGMY primers and the Linear array HPV genotyping test. J Clin Microbiol, 44, 1998-2006.

Cribier B, Worret WI, Braun-Falco M, et al (2006). Expression patterns of hair and epithelial keratins and transcription factors $H O X C 13$, LEF1, and beta-catenin in a malignant pilomatricoma: a histological and immunohistochemical study. J Cutan Pathol, 33, 1-9.

Chen JL, Li J, Kiriluk KJ, et al (2012). Deregulation of a $H O X$ protein regulatory network spanning prostate cancer initiation and progression. Clin Cancer Res, 18, 4291-302.

Duluc I, Lorentz O, Fritsch C, et al (1997). Changing intestinal connective tissue interactions alters homeobox gene expression in epithelial cells. J Cell Sci, 110, 1317-24.

Ferlay J, Soerjomataram I, Dikshit R, et al (2015). Cancer incidence and mortality worldwide: sources, methods and major patterns in GLOBOCAN 2012. Int J Cancer, 136, 359-86.

Gilbert PM, Mouw JK, Unger MA, et al (2010). HOXA9 regulates BRCA1 expression to modulate human breast tumor phenotype. J Clin Invest, 120, 1535-50.

Gonzalez-Herrera A, Salgado-Bernabe M, Velazquez-Velazquez $\mathrm{C}$, et al (2015). Increased expression of HOXB2 and $H O X \mathrm{~B} 13$ proteins is associated with HPV infection and cervical cancer progression. Asian Pac J Cancer Prev, 16, 1349-53.

Gravitt PE, Peyton CL, Alessi TQ, et al (2000). Improved amplification of genital human papillomaviruses. J Clin Microbiol, 38, 357-61.

Guerrero-Preston R, Michailidi C, Marchionni L, et al (2014). Key tumor suppressor genes inactivated by "greater promoter" methylation and somatic mutations in head and neck cancer. Epigenetics, 9, 1031-46.

Guerrero-Preston R, Soudry E, Acero J, et al (2011). NID2 and HOXA9 promoter hypermethylation as biomarkers for prevention and early detection in oral cavity squamous cell carcinoma tissues and saliva. Cancer Prev Res (Phila), 4, 1061-72.

Hsieh-Li HM, Witte DP, Weinstein M, et al (1995). HOXA 11 structure, extensive antisense transcription, and function in male and female fertility. Development, 121, 1373-85.

Hung YC, Ueda M, Terai Y, et al (2003). Homeobox gene expression and mutation in cervical carcinoma cells. Cancer Sci, 94, 437-41.

Hwang JA, Lee BB, Kim Y, et al (2015). HOXA9 inhibits migration of lung cancer cells and its hypermethylation is associated with recurrence in non-small cell lung cancer. Mol Carcinog, 54, 72-80.

Hyland PL, McDade SS, McCloskey R, et al (2011). Evidence for alteration of EZH2, BMI1, and KDM6A and epigenetic reprogramming in human papillomavirus type 16 E6/E7expressing keratinocytes. J Virol, 85, 10999-1006.

Jave-Suarez LF, Winter H, Langbein L, et al (2002). HOXC13 is involved in the regulation of human hair keratin gene expression. J Biol Chem, 277, 3718-26.

Karamboulas C, Ailles L (2013). Developmental signaling pathways in cancer stem cells of solid tumors. Biochim Biophys Acta, 1830, 2481-95. 
Kasiri S, Ansari KI, Hussain I, et al (2013). Antisense oligonucleotide mediated knockdown of $\mathrm{HOXC13}$ affects cell growth and induces apoptosis in tumor cells and over expression of $H O X C 13$ induces 3D-colony formation. RSC $A d v, 3,3260-9$.

Kim YJ, Yoon HY, Kim JS, et al (2013). HOXA9, ISL1 and ALDH1A3 methylation patterns as prognostic markers for nonmuscle invasive bladder cancer: array-based DNA methylation and expression profiling. Int J Cancer, 133, 1135-42.

Ko SY, Naora H (2014). HOXA9 promotes homotypic and heterotypic cell interactions that facilitate ovarian cancer dissemination via its induction of P-cadherin. Mol Cancer, 13, 170 .

Lappin TR, Grier DG, Thompson A, et al (2006). HOX genes: seductive science, mysterious mechanisms. Ulster Med J, $\mathbf{7 5}, 23-31$.

Li LC, Dahiya R (2002). MethPrimer: designing primers for methylation PCRs. Bioinformatics, 18, 1427-31.

Lopez R, Garrido E, Pina P, et al (2006a). HOXB homeobox gene expression in cervical carcinoma. Int J Gynecol Cancer, 16, 329-35.

Lopez R, Garrido E, Vazquez G, et al (2006b). A subgroup of $H O X$ Abd-B gene is differentially expressed in cervical cancer. Int J Gynecol Cancer, 16, 1289-96.

Marcinkiewicz KM, Gudas LJ (2014a). Altered epigenetic regulation of homeobox genes in human oral squamous cell carcinoma cells. Exp Cell Res, 320, 128-43.

Marcinkiewicz KM, Gudas LJ (2014b). Altered histone mark deposition and DNA methylation at homeobox genes in human oral squamous cell carcinoma. J Cell Physiol, 229, 1405-16.

Mehrazarin S, Chen W, Oh JE, et al (2015). The p63 Gene Is Regulated by Grainyhead-like 2 (GRHL2) through Reciprocal Feedback and Determines the Epithelial Phenotype in Human Keratinocytes. J Biol Chem, 290, 19999-20008.

Milne TA, Kim J, Wang GG, et al (2010). Multiple interactions recruit MLL1 and MLL1 fusion proteins to the HOXA9 locus in leukemogenesis. Mol Cell, 38, 853-63.

Perez-Plasencia C, Duenas-Gonzalez A, Alatorre-Tavera B (2008). Second hit in cervical carcinogenesis process: involvement of wnt/beta catenin pathway. Int Arch Med, 1,10 .

Qi Z, Xu X, Zhang B, et al (2010). Effect of simultaneous silencing of HPV-18 E6 and E7 on inducing apoptosis in HeLa cells. Biochem Cell Biol, 88, 697-704.

Raines AM, Adam M, Magella B, et al (2013). Recombineeringbased dissection of flanking and paralogous $H O X$ gene functions in mouse reproductive tracts. Development, 140, 2942-52.

Rauch T, Wang Z, Zhang X, et al (2007). Homeobox gene methylation in lung cancer studied by genome-wide analysis with a microarray-based methylated $\mathrm{CpG}$ island recovery assay. Proc Natl Acad Sci USA, 104, 5527-32.

Rossig L, Urbich C, Bruhl T, et al (2005). Histone deacetylase activity is essential for the expression of HOXA9 and for endothelial commitment of progenitor cells. J Exp Med, 201, 1825-35.

Sasagawa T, Takagi H, Makinoda S (2012). Immune responses against human papillomavirus (HPV) infection and evasion of host defense in cervical cancer. J Infect Chemother, 18, 807-15.

Shah N, Sukumar S (2010). The HOX genes and their roles in oncogenesis. Nat Rev Cancer, 10, 361-71.

Sun X, Liu B, Ji W, et al (2013). The role of HOXA9 in human laryngeal squamous cell carcinoma. Oncol Res, 20, 467-72.
Svingen T, Tonissen KF (2006). HOX transcription factors and their elusive mammalian gene targets. Heredity (Edinb), 97, 88-96.

Torres-Reyes LA, Alvarado-Ruiz L, Pina-Sanchez P, et al (2014). Expression of transcription factor grainyhead-like 2 is diminished in cervical cancer. Int J Clin Exp Pathol, 7, 7409-18.

Uchida K, Veeramachaneni R, Huey B, et al (2014). Investigation of HOXA9 promoter methylation as a biomarker to distinguish oral cancer patients at low risk of neck metastasis. BMC Cancer, 14, 353.

Wu Q, Lothe RA, Ahlquist T, et al (2007). DNA methylation profiling of ovarian carcinomas and their in vitro models identifies HOXA9, HOXB5, SCGB3A1, and CRABP1 as novel targets. Mol Cancer, $6,45$.

Zhu JY, Sun QK, Wang W, et al (2014). High-level expression of $H O X \mathrm{~B} 13$ is closely associated with tumor angiogenesis and poor prognosis of hepatocellular carcinoma. Int J Clin Exp Pathol, 7, 2925-33. 